\title{
Micropropagation and essential oil characterization of Plectranthus amboinicus (Lour.) Sprengel, an aromatic medicinal plant
}

\begin{abstract}
Plectranthus amboinicus (Loureiro) Sprengel is a therapeutically valued herb highly sought by various industries. This has led to its uncontrolled harvest from the wild leading to the risk of extinction. As an alternative, commercial propagation of $\mathrm{P}$. amboinicus is desirable to avoid further depletion of wild populations. This paper describes the establishment of an efficient protocol for the multiplication, rooting and acclimatization of P. amboinicus. The greatest frequency of shoot multiplication of P. amboinicus was on semi-solid Murashige and Skoog (MS) medium supplemented with $0.4 \mathrm{mg}$ L-1 6-benzylaminopurine (BAP). Media supplemented with higher concentrations of BAP or used in combination with gibberellic acid (GA3) produced abnormal plantlets. Optimal rooting was observed on plantlets cultured in half strength MS medium. Acclimatization was achieved by transferring the rooted plants onto sterile peat-moss moistened with MS medium and exposed to the glasshouse environment gradually over a period of 2 months. P. amboinicus responded readily to tissue culture, but careful attention was required to media formulation to avoid abnormal plantlet development. The composition of essential oils extracted from field grown plants and in vitro microshoots were comparable.
\end{abstract}

Keyword: Medicinal plant; Micropropagation; Plant growth regulators; Essential oils; GC-MS 